\title{
Hidratação de ligantes na presença de matriz e aditivos
}

\section{(Hydration of binders in the presence of matrix and additives)}

\author{
I. R. Oliveira, V. C. Pandolfelli \\ Departamento de Engenharia de Materiais, Universidade Federal de S. Carlos \\ Rod. Washington Luiz, km 235, C.P. 676, S. Carlos, SP 13565-905 \\ ivone@iris.ufscar.br,vicpando@power.ufscar.br
}

\begin{abstract}
Resumo
Ligantes hidráulicos, como os cimentos de aluminato de cálcio e as aluminas hidratáveis, são os responsáveis pelo desenvolvimento de resistência mecânica de concretos refratários (desmoldagem) influenciando também as suas propriedades reológicas (trabalhabilidade). Tal influencia se deve ao processo de hidratação desses materiais cujo controle depende do conhecimento do efeito das diferentes variáveis na cinética desse processo. A adição de matriz ao cimento influencia o seu comportamento de hidratação, bem como a extensão do período de indução, a composição das fases e dos produtos de hidratação. Aditivos podem atuar como retardadores dificultando o processo de dissolução do cimento, por meio da formação de barreiras insolúveis ao redor das suas partículas e favorecendo a formação de hidratos mais solúveis. Podem também atuar como aceleradores favorecendo a formação de hidratos menos solúveis e pela formação de núcleos iniciadores do processo de crescimento dos cristais dos hidratos. Assim, neste trabalho foi estudada a influência de diferentes tipos de matriz e aditivos no processo de hidratação de diferentes ligantes hidráulicos.
\end{abstract}

Palavras-chave: hidratação, aluminato de cálcio, alumina hidratável, matriz, aditivos.

\section{Abstract}

Hydraulic binders such as hydratable alumina and calcium aluminate cement present a major influence on the rheological properties and mechanical strength development of castables, defining their workability and demoulding times, respectively. Such behavior is related to the hydration process and its control depends on the knowledge of the variables that determine the kinetics of the involved reactions. The matrix addition to cement influences the hydration behaviour, as well as, the length of the induction period, the composition of the phases and of the hydration products. Additives such as the retarders can inhibit the process of cement dissolution, by the build up of insoluble barriers around the cement particles, and enhance the formation of more soluble hydrates. They can also behave as accelerators inducing the formation of less soluble hydrates and speeding up the beginning of the hydrate nucleation. In this context, the objective of this work was to evaluate the influence of different kind of matrix and additives on the hydration process of different sources of the hydraulic binder.

Keywords: binders, calcium aluminate cement, hydratable alumina, matrix, additives.

\section{INTRODUÇÃO}

Ligantes hidráulicos são, em sua maioria, óxidos capazes de se rehidratar promovendo a coesão das partículas do concreto refratário conformado. Além do desenvolvimento de resistência mecânica, determinante do tempo de desmoldagem, os ligantes também influenciam as propriedades reológicas desses materiais, controlando sua trabalhabilidade. Nesta categoria, destacam-se os cimentos de alta alumina, também conhecidos como cimentos de aluminato de cálcio (CAC).

$\mathrm{O}$ processo de hidratação do $\mathrm{CAC}$ inicia-se quando a água entra em contato com a superfície das partículas do cimento:

$$
\mathrm{Ca}\left(\mathrm{AlO}_{2}\right)_{2}+4 \mathrm{H}_{2} \mathrm{O} \Leftrightarrow \mathrm{Ca}^{2+}+2 \mathrm{Al}(\mathrm{OH})_{4}^{-}
$$

Os íons $\mathrm{Al}(\mathrm{OH})_{4}^{-}$comportam-se como uma base, sendo que uma pequena parte deles se dissocia em íons $\mathrm{Al}^{3+} \mathrm{e} \mathrm{OH}^{-}$ em solução, estabelecendo um equilíbrio comandado pela constante básica $\mathrm{K}_{\mathrm{b}}$. Este fenômeno é o responsável pelo aumento do $\mathrm{pH}$ do meio:

$$
\mathrm{Al}(\mathrm{OH})_{4}^{-} \Leftrightarrow \mathrm{Al}^{3+}+4 \mathrm{OH}^{-} \quad \mathrm{K}_{\mathrm{b}}=1,8 \times 10^{2}
$$

A ocorrência destas reações químicas provoca a dissolução das fases anidras que compõem o cimento proporcionando um aumento das concentrações dos íons $\mathrm{Ca}^{2+} \mathrm{e} \mathrm{Al}(\mathrm{OH})_{4}{ }^{-}$em solução. Este processo continua até que um estado de saturação da solução, em relação à dissolução do cimento, seja alcançado. Desta forma, o processo atinge um estado de equilíbrio químico e a dissolução do cimento é interrompida. Entretanto, em relação aos produtos da reação de hidratação, o equilíbrio alcançado não é estável, ou seja, a solução está supersaturada em relação aos compostos formados e, por esse motivo, existe uma tendência natural 
para que ocorra a precipitação desses produtos [1].

Apesar de favorecida, a precipitação dos hidratos envolve um processo lento de nucleação, durante o qual não ocorre precipitação. Esse tempo de espera para que se atinjam condições ideais para a precipitação é conhecido como "período de indução". Este estado de equilíbrio metaestável é mantido até que se vença a barreira de energia de ativação $\left(\mathrm{E}_{\mathrm{a}}\right)$ necessária à formação dos primeiros germes dos cristais dos hidratos [1].

A partir da formação destes germes de hidratos a velocidade do processo não é mais limitada pela $\mathrm{E}_{\mathrm{a}}$, uma vez que a precipitação passa a ocorrer por meio do crescimento deles. Dessa maneira, íons de $\mathrm{Ca}^{2+} \mathrm{e} \mathrm{Al}(\mathrm{OH})_{4}^{-}$são retirados da solução, proporcionando a retomada da dissolução das partículas de cimento, o que possibilita um contínuo crescimento dos hidratos [1].

Os cimentos aluminosos comerciais $\left(70 \% \mathrm{de} \mathrm{Al}_{2} \mathrm{O}_{3}\right)$ são constituídos principalmente pelas fases: $\mathrm{CaO} \cdot \mathrm{Al}_{2} \mathrm{O}_{3}(\mathrm{CA})$, cerca de 40 a $70 \%$ do produto; $\mathrm{CaO} \cdot 2 \mathrm{Al}_{2} \mathrm{O}_{3}\left(\mathrm{CA}_{2}\right)$, que é a segunda em proporção $(<25 \%)$ e a fase $12 \mathrm{CaO} \cdot 7 \mathrm{Al}_{2} \mathrm{O}_{3}$ $\left(\mathrm{C}_{12} \mathrm{~A}_{7}\right)$, em teores de $3 \%$ ou menos. A fase $\mathrm{CA}_{2}$ é a mais refratária das três e devido a sua relação $\mathrm{CaO} / \mathrm{Al}_{2} \mathrm{O}_{3}$ requer um longo tempo para completar sua hidratação. Já a fase $\mathrm{C}_{12} \mathrm{~A}_{7}$, ao contrário, possui baixa refratariedade, entretanto, necessita de um tempo curto para se hidratar e pode acelerar o tempo de pega da fase CA [2]. Dessa forma, quanto maior o teor da fase $\mathrm{C}_{12} \mathrm{~A}_{7}$ presente no cimento, mais rápido ocorrerá a saturação e a precipitação de hidratos. Além disso, a diferença imposta pelo processo de hidratação das diferentes fases anidras está relacionada com a quantidade relativa entre as concentrações de $\mathrm{Ca}^{2+}$ e $\mathrm{Al}(\mathrm{OH})_{4}^{-}$que cada uma delas pode gerar em solução.

Consequentemente, a concentração dos íons $\mathrm{Ca}^{2+} \mathrm{e}$ $\mathrm{Al}(\mathrm{OH})_{4}^{-}$em solução permitirá e ditará a fase de hidrato que será formada. A relação entre as concentrações desses íons em solução é convencionalmente representada por C/A que expressa a quantidade de cálcio e de alumínio na forma de $\mathrm{CaO}$ e $\mathrm{Al}_{2} \mathrm{O}_{3}$, respectivamente.

Sendo assim, o valor de C/A determina qual hidrato será formado em uma determinada temperatura. Para a temperatura de $25{ }^{\circ} \mathrm{C}$ e relação entre $\left[\mathrm{Ca}^{2+}\right]$ e $\left[\mathrm{Al}(\mathrm{OH})_{4}{ }^{-}\right]$ próximas a 1, a formação do hidrato $\mathrm{CAH}_{10}$ é favorecida. Com o aumento dessa relação, ou seja, para maiores teores de $\mathrm{Ca}^{2+}$, passa-se a favorecer a formação de $\mathrm{C}_{2} \mathrm{AH}_{8}$. Já para maiores concentrações de $\mathrm{Al}(\mathrm{OH})_{4}^{-}$é predominante a formação de $\mathrm{AH}_{3}$.

Esses tipos de hidratos formados a $25^{\circ} \mathrm{C}$ diferem quanto ao grau de solubilidade, na seguinte ordem $\mathrm{C}_{2} \mathrm{AH}_{8}<\mathrm{CAH}_{10}$ $<\mathrm{AH}_{3}$. A formação do tipo menos solúvel implica numa alta velocidade de precipitação reduzindo o tempo de pega do cimento. Um raciocínio semelhante pode ser aplicado para se entender o atraso no tempo de pega quando o hidrato favorecido é o $\mathrm{AH}_{3}$, que inicialmente tem uma solubilidade maior que $\mathrm{CAH}_{10}$ e por esse motivo requer um maior período de indução.

A temperatura também determina o tipo de hidrato formado. Em baixas temperaturas $\left(5-10{ }^{\circ} \mathrm{C}\right)$, a fase majoritária formada é o hidrato $\mathrm{CAH}_{10}\left(\mathrm{CaO} \mathrm{Al}_{2} \mathrm{O}_{3} \cdot 10 \mathrm{H}_{2} \mathrm{O}\right)$ que é o menos denso $\left(1,72 \mathrm{~g} \cdot \mathrm{cm}^{-3}\right)$ e o mais solúvel. $\mathrm{O} \mathrm{C}_{2} \mathrm{AH}_{8}$ $\left(2 \mathrm{CaO} . \mathrm{Al}_{2} \mathrm{O}_{3} \cdot 8 \mathrm{H}_{2} \mathrm{O}\right)$ forma-se entre $22-35^{\circ} \mathrm{C}$ e possui valores intermediários de densidade $\left(1,95 \mathrm{~g} . \mathrm{cm}^{-3}\right)$ e de solubilidade. Já o hidrato $\mathrm{C}_{3} \mathrm{AH}_{6}\left(3 \mathrm{CaO} \cdot \mathrm{Al}_{2} \mathrm{O}_{3} \cdot 6 \mathrm{H}_{2} \mathrm{O}\right)$ é aquele formado majoritariamente em temperaturas acima de $35{ }^{\circ} \mathrm{C}$, sendo o mais denso $\left(2,52{\mathrm{~g} . \mathrm{cm}^{-3}}^{-3}\right)$ e o menos solúvel dos três $[1,3]$.

Um outro tipo de ligante utilizado é a alumina hidratável a qual pode ser composta por uma variedade de fases de transição da alumina, principalmente a alumina-rho ( $\rho$ ), que apresenta baixa cristalinidade e alta área superficial. A capacidade ligante deste material deriva da sua fácil rehidratação quando em contato com a água a temperatura ambiente [4]:

$\rho-\mathrm{Al}_{2} \mathrm{O}_{3}+\mathrm{H}_{2} \mathrm{O} \rightarrow \mathrm{Al}_{2} \mathrm{O}_{3} \cdot 3 \mathrm{H}_{2} \mathrm{O}+\mathrm{Al}_{2} \mathrm{O}_{3}(1 \approx 2) \mathrm{H}_{2} \mathrm{O}$

Nos primeiros estágios da hidratação da alumina $\rho$, uma espessa camada de alumina gel é formada e, com o decorrer da reação, parte deste gel é transformado em boemita $\left(\mathrm{Al}_{2} \mathrm{O}_{3} \cdot 1 \approx 2 \mathrm{H}_{2} \mathrm{O}\right)$ e principalmente baierita $\left(\mathrm{Al}_{2} \mathrm{O}_{3} \cdot 3 \mathrm{H}_{2} \mathrm{O}\right)$. Os cristais interligados de baierita e o gel conferem resistência mecânica a verde aos refratários por meio do preenchimento de poros e redução dos defeitos superficiais. Tal cristalização também favorece a ligação entre os agregados, unindo os grãos adjacentes à matriz circundante [5].

A cinética do processo de hidratação desses ligantes pode ser significativamente modificada em função do tipo de ligante hidráulico e da presença de materiais finos (matriz) e aditivos. Neste contexto, esse trabalho teve como objetivo avaliar a influência dessas variáveis no processo de hidratação por meio de medidas de temperatura e ensaios reológicos oscilatórios e de força normal em função do tempo.

\section{MATERIAIS E MÉTODOS}

Os ligantes hidráulicos avaliados foram os cimentos de aluminato de cálcio CA14M e CA270 (Almatis-EUA), Secar 71 e Secar Plenium (Lafarge Aluminates-França) e a alumina hidratável Alphabond 300 (Almatis-EUA), cujas características são apresentadas na Tabela I. As aluminas utilizadas (Tabela II) foram o Esy Pump 1000 e as aluminas calcinadas A17-NE e A-1000SG (Almatis-EUA). Tais aluminas normalmente constituem as matrizes de concretos refratários sendo utilizadas isoladamente (Esy Pump ou A17-NE) ou combinadas A17-NE:A1000SG (93:7\%). Como aditivo inorgânico foi selecionado o carbonato de lítio ( $\mathrm{Li}_{2} \mathrm{CO}_{3}$, Labsynth-Brasil). Já como dispersantes foram avaliados diferentes compostos: polimetacrilato de sódio (Darvan-7S, Vanderbilt, $2500 \mathrm{~g} / \mathrm{mol}$ ), ácido cítrico anidro (Labsynth, $192 \mathrm{~g} / \mathrm{mol}$ ), citrato de diamônio (Sigma Aldrich), hexametafosfato de sódio (Labsynth) e os polímeros da família do poliglicol, fornecidos pela SKW Polymers (CV, CT, CQ e CS).

Para verificar a influência da matriz no processo de hidratação foram preparadas suspensões aquosas dos diferentes ligantes hidráulicos contendo $50 \%$ em peso dos 
Tabela I - Composições químicas e propriedades físicas dos ligantes estudados.

[Table I - Chemical compositions and physical properties of the binders analyzed.]

\begin{tabular}{|c|c|c|c|c|c|}
\hline Fases $(\%)$ & $\begin{array}{c}\mathrm{CA} \\
14 \mathrm{M}\end{array}$ & $\begin{array}{l}\text { CA } \\
270\end{array}$ & $\begin{array}{c}\text { Secar } \\
71\end{array}$ & $\begin{array}{c}\text { Secar } \\
\text { Plenium }\end{array}$ & $\begin{array}{c}\text { Alphabonc } \\
300\end{array}$ \\
\hline $\mathrm{Al}_{2} \mathrm{O}_{3}$ & 72 & 73 & 68 & 82 & 88 \\
\hline $\mathrm{CaO}^{2}$ & 27 & 26 & 31 & 18 & 0,1 \\
\hline $\mathrm{SiO}_{2}$ & 0,3 & 0,3 & 0,8 & 0,3 & 0,3 \\
\hline $\mathrm{Na}_{2} \mathrm{O}$ & 0,3 & 0,3 & 0,5 & 0,7 & 0,6 \\
\hline $25-250^{\circ} \mathrm{C}^{*}$ & - & - & - & - & 4,5 \\
\hline $250-1100^{\circ} \mathrm{C}^{*}$ & - & - & - & - & 6,5 \\
\hline $\begin{array}{c}\text { Densidade } \\
\left(\mathrm{g} / \mathrm{cm}^{3}\right)\end{array}$ & 2,96 & 3,15 & 2,98 & 3,25 & 3,20 \\
\hline $\begin{array}{l}\text { ASE BET } \\
\left(\mathrm{m}^{2} / \mathrm{g}\right)\end{array}$ & 1,87 & 1,88 & 1,17 & 5,78 & 194 \\
\hline $\mathrm{D}_{50}(\mu \mathrm{m})$ & 9,4 & 7,8 & 13 & 10 & 3,3 \\
\hline
\end{tabular}

* Perda de massa no intervalo de temperatura.

Tabela II - Composições químicas e propriedades físicas dos materiais finos utilizados.

[Table II - Chemical compositions and physical properties of the fines materials tested.]

\begin{tabular}{cccc}
\hline Fases (\%) & $\begin{array}{c}\text { Esy Pump } \\
1000\end{array}$ & A17-NE* & A-1000SG \\
\hline $\mathrm{Al}_{2} \mathrm{O}_{3}$ & 99,4 & 99,8 & 99,8 \\
$\mathrm{CaO}$ & - & 0,02 & 0,02 \\
$\mathrm{SiO}{ }_{2}$ & 0,10 & 0,03 & 0,03 \\
$\mathrm{Na}_{2} \mathrm{O}$ & 0,30 & 0,10 & 0,07 \\
Densidade $\left(\mathrm{g} / \mathrm{cm}^{3}\right)$ & 3,99 & 3,94 & 3,95 \\
$\mathrm{ASE} \mathrm{BET}\left(\mathrm{m}^{2} / \mathrm{g}\right)$ & 3,3 & 2,9 & 8,4 \\
$\mathrm{D}_{50}(\mu \mathrm{m})$ & 2,6 & 4,0 & 0,57 \\
\hline
\end{tabular}

*A matriz A17-NE:A-1000SG (93:7) apresenta ASE $=3,28 \mathrm{~m}^{2} / \mathrm{g}$ e teor de $\mathrm{Na}_{2} \mathrm{O}$ de $0,098 \%$.

materiais finos, resultando numa concentração de sólidos de $26 \%$ em volume. Já para avaliar a influência dos aditivos dispersantes foram preparadas suspensões aquosas dos diferentes ligantes (16,8\%-v. ou 37-39\%-p de sólidos) contendo os aditivos dispersantes $\left(0,1 ; 0,3\right.$ ou $0,9 \mathrm{mg} / \mathrm{m}^{2}$ em relação ao material). Para ambas as avaliações (matriz e dispersante) também foi verificada a influência da adição simultânea de $\mathrm{Li}_{2} \mathrm{CO}_{3}(0,01 \%$-p sobre a massa de cimento com exceção do Secar Plenium (0,02\%-p) e Alphabond $300(0,32 \%$-p) a fim de manter a mesma relação massa dispersante/massa $\mathrm{Li}_{2} \mathrm{CO}_{3}$ ).

Imediatamente após a mistura com auxílio de um agitador mecânico, as suspensões foram submetidas a medidas de temperatura.

As medidas de temperatura foram monitoradas em função do tempo, com o auxílio de um termopar conectado a um sistema automático de aquisição de dados (ESA 9800, Matec Applied Sciences - UK). Esse sensor foi inserido nas suspensões de cimento ou cimento-matriz sendo as medidas imediatamente iniciadas. O sistema para o controle de temperatura foi especialmente desenvolvido para permitir medidas na temperatura desejada $\left(50{ }^{\circ} \mathrm{C}\right)$.

Adicionalmente, foi avaliada a influência das combinações matriz-ácido cítrico, matriz-carbonato de lítio e matriz-ácido cítrico-carbonato de lítio por meio de ensaios reológicos oscilatórios e de força normal, com controle de temperatura em $50^{\circ} \mathrm{C}$.

Suspensões aquosas dos diferentes tipos de ligantes foram preparadas na presença de 50\% em peso de Esy Pump para a realização das medidas na ausência ou na presença de ácido cítrico $\left(0,1 \mathrm{mg} / \mathrm{m}^{2}\right)$ e $\mathrm{Li}_{2} \mathrm{CO}_{3}(0,01 \%$-p sobre a massa de cimento com exceção do Secar Plenium $(0,02 \%$-p) e Alphabond $300(0,32 \%$-p) ) quando puros ou combinados.

Os ensaios reológicos oscilatórios e de força normal em função do tempo foram realizados utilizando-se o equipamento Rheostress 300 da ThermoHaake. Uma das medidas obtidas nos ensaios reológicos é o módulo de armazenamento (G'), que representa às características elásticas da suspensão [6]. A ferramenta de medição utilizada foi a vane, a qual oferece vantagens como a eliminação de efeitos de parede, mínima ruptura da estrutura do material sob carregamento, facilidade de preparação de amostra e alta velocidade de análise. Esta ferramenta possui geometria multifacetada e quando imersa em uma amostra é rotacionada, provocando cisalhamento [7, 8].

Para os ensaios de força normal adotou-se uma metodologia desenvolvida por Anjos et al [9]. Neste método, a suspensão a ser analisada é dividida em vários recipientes. Tais amostras são cobertas com óleo para evitar a secagem e mantidas em temperatura constante em uma câmara climática (Vötsch, modelo 20-20). Em intervalos de tempo regulares, as amostras são, uma a uma, submetidas às medidas de força normal (cada amostra é ensaiada em um tempo de espera diferente após a mistura da suspensão). A ferramenta, um indentador em forma de lâmina, move-se com taxa de penetração previamente ajustada a fim de evitar a formação de trincas nas amostras. Os resultados obtidos de força normal em função do tempo de coleta para cada amostra foram derivados em função do tempo, gerando uma taxa de força normal.

\section{RESULTADOS E DISCUSSÃO}

Neste trabalho, medidas de temperatura em função do tempo foram realizadas para suspensões dos diferentes tipos de ligantes a fim de avaliar a influência da matriz e de aditivos dispersantes quando puros ou combinados com carbonato de lítio. O procedimento utilizado para determinação dos tempos de pega a partir desses ensaios foi descrito anteriormente [10].

Medidas de temperatura das suspensões de ligante têm se mostrado muito útil para o acompanhamento das reações de hidratação desses materiais. O aumento de temperatura é característico das reações de hidratação dos ligantes 
constituindo-se numa técnica muito simples. Por meio da introdução de um termopar na pasta de ligante esse aumento de temperatura pode ser detectado e usado como medida do tempo no qual ocorrem as suas reações de hidratação e conseqüente endurecimento.

\section{Influência da matriz e aditivo inorgânico}

A influência isolada de matriz e aditivo inorgânico $\left(\mathrm{Li}_{2} \mathrm{CO}_{3}\right)$ no tempo de pega dos diferentes ligantes foi estudada anteriormente [10]. Neste trabalho foi verificado encurtamento do período de indução e conseqüente decréscimo do tempo de pega para a mistura de todos os tipos de ligantes com as diferentes matrizes, com exceção do Secar Plenium e Alphabond 300 (Tabela III). Tal influencia foi inicialmente correlacionada com a área superficial específica (ASE) da matriz uma vez que quanto maior a ASE da matriz mais pronunciado é o encurtamento do período de indução durante o processo de hidratação [11]. Entretanto, quando comparado a ASE das diferentes matrizes avaliadas (Tabela II) observou-se valores bastante próximos indicando que alguma outra diferença característica das diferentes matrizes deveria ser predominante. De fato, foi observada uma influencia predominante do teor $\mathrm{de}_{2} \mathrm{O}$ das matrizes no tempo de indução.

A matriz Esy Pump possui o maior teor deste óxido (Tabela II), resultando nos menores valores de tempo de pega. Isso pode ser entendido visto que o sódio liberado em solução pode atuar diminuindo a quantidade de íons $\mathrm{Al}(\mathrm{OH})_{4}^{-}$ por meio da formação de $\mathrm{NaAl}(\mathrm{OH})_{4}$, causando assim um aumento da relação C/A o que favorece a precipitação do hidrato menos solúvel. A presença de um maior teor de $\mathrm{Na}^{+}$ em solução, quando utilizado a Esy Pump, foi confirmada anteriormente pelo significativo aumento de condutividade quanto comparado às demais matrizes [10].

No caso do Secar Plenium somente foi verificado decréscimo do tempo de pega para o sistema mais rico em sódio, ou seja, na presença de Esy Pump. Isso porque o decréscimo pronunciado na quantidade íons $\mathrm{Al}(\mathrm{OH})_{4}$ - deve ter resultado em uma quantidade relativa entre as concentrações de $\mathrm{Ca}^{2+} \mathrm{e} \mathrm{Al}(\mathrm{OH})_{4}^{-}$tal que favoreceu a formação do hidrato menos solúvel $\mathrm{C}_{3} \mathrm{AH}_{6}$, uma vez que a temperatura de ensaio foi de $50{ }^{\circ} \mathrm{C}$. Já para a alumina hidratável Alphabond 300 não foi observada uma influência significativa da matriz dado o seu tempo de reação já ser bastante curto.

Quanto à influência do $\mathrm{Li}_{2} \mathrm{CO}_{3}$, observou-se uma considerável redução no tempo de pega na presença deste aditivo uma vez que os íons $\mathrm{Li}^{+}$proporcionam a formação de compostos insolúveis do tipo $\operatorname{LiAl}(\mathrm{OH})_{4}$, retirando íons $\mathrm{Al}(\mathrm{OH})_{4}^{-}$da solução $[12,13]$. Dessa forma, os sais de lítio também atuam na reação de hidratação do cimento favorecendo a formação de hidratos menos solúveis que irão precipitar mais facilmente.

Além disso, a precipitação desses compostos insolúveis de lítio é mais rápida do que a precipitação dos hidratos de aluminato de cálcio e apresentam estrutura cristalina necessária para servir de germes para a nucleação do hidrato $\mathrm{C}_{2} \mathrm{AH}_{8}$, independente da temperatura. Isso faz com que a barreira de energia necessária à nucleação desse hidrato deixe de existir.

Tais fenômenos puderam explicar a considerável redução no tempo de pega observada para os cimentos CA14M, CA270 e Secar 71. Entretanto, o efeito acelerador do carbonato de lítio foi menos pronunciado para o Secar Plenium. $\mathrm{O}$ fato deste tipo de cimento apresentar um menor teor de $\mathrm{CaO}$ (Tabela I) faz com que se tenha menos íons $\mathrm{Ca}^{2+}$ provenientes da dissolução da fase anidra resultando em uma menor tendência a formação do hidrato $\mathrm{C}_{2} \mathrm{AH}_{8}$, que é aquele favorecido quando na presença de $\mathrm{Li}_{2} \mathrm{CO}_{3}$. Dessa forma, foi concluído que não basta existir germes que facilitem a precipitação de um determinado hidrato, mas deve também haver uma concentração de íons em solução suficientes para a sua formação.

No presente trabalho também é apresentada a influência conjunta de matriz e $\mathrm{Li}_{2} \mathrm{CO}_{3}$ para os diferentes tipos de ligantes, em comparação a atuação isolada de matriz e $\mathrm{Li}_{2} \mathrm{CO}_{3}$ (Tabela III).

Quando comparado o efeito da adição de $\mathrm{Li}_{2} \mathrm{CO}_{3}$ sobre o

Tabela III - Influência do $\mathrm{Li}_{2} \mathrm{CO}_{3}$, da matriz e da matriz$\mathrm{Li}_{2} \mathrm{CO}_{3}$ no tempo de pega (minutos) de diferentes tipos de ligantes hidráulicos avaliada por meio de ensaios de temperatura.

[Table III - Influence of $\mathrm{Li}_{2} \mathrm{CO}_{3}$, matrix and matrix- $\mathrm{Li}_{2} \mathrm{CO}_{3}$ on the setting time (minutes) of different kind of hydraulic binder evaluated through temperature measurements.]

\section{CA14M CA270 Secar Secar Alphabond}

71 Plenium 300

\begin{tabular}{crrrrc}
\hline Puro & 60 & 51 & 94 & 81 & 11 \\
$\mathrm{Li}_{2} \mathrm{CO}_{3}$ & 12 & 13 & 11 & 62 & 3 \\
$\mathrm{~A} 17 \mathrm{NE} / \mathrm{A}_{1000}$ & 48 & 38 & 47 & 80 & 13 \\
$\mathrm{~A} 17 / \mathrm{A}_{1000, \mathrm{Li}_{2} \mathrm{CO}_{3}} 28$ & 24 & 26 & 78 & 14 \\
$\mathrm{~A} 17 \mathrm{NE}$ & 44 & 32 & 50 & 85 & 14 \\
$\mathrm{~A} 17 \mathrm{NE}, \mathrm{Li}_{2} \mathrm{CO}_{3}$ & 15 & 20 & 18 & 89 & 14 \\
Esy Pump & 29 & 25 & 28 & 60 & 13 \\
Esy Pump, $\mathrm{Li}_{2} \mathrm{CO}_{3}$ & 10 & 19 & 13 & 54 & 12 \\
\hline
\end{tabular}

cimento puro, com a adição na suspensão de cimento combinado com a matriz, observa-se que seu efeito é reduzido na presença de matriz. Esse comportamento pode ser entendido considerandose que, quando se adiciona $\mathrm{Li}^{+}$no sistema cimento/matriz o aumento na razão entre $\left[\mathrm{Ca}^{2+}\right]$ e $\left[\mathrm{Al}(\mathrm{OH})_{4}^{-}\right]$é menor que aquele observado para o caso do cimento puro (considerando os íons $\mathrm{Al}(\mathrm{OH})_{4}^{-}$provenientes da alumina). Além disso, nessa situação, verifica-se a presença de sítios de nucleação tanto para o $\mathrm{C}_{2} \mathrm{AH}_{8}$ (formados pela precipitação do $\mathrm{LiAl}(\mathrm{OH})_{4}^{-}$) quanto para o $\mathrm{AH}_{3}$ (devido a presença da matriz). Entretanto, como a razão entre as concentrações dos íons provenientes da dissolução do cimento não aumenta como no caso da adição de $\mathrm{Li}^{+}$na pasta de cimento puro, a formação do $\mathrm{AH}_{3}$ deve prevalecer. Dessa forma, a menor redução observada no tempo de pega causada pela adição de $\mathrm{Li}_{2} \mathrm{CO}_{3}$ na suspensão cimento/matriz pode ser atribuída a não predominância na formação do hidrato menos solúvel $\left(\mathrm{C}_{2} \mathrm{AH}_{8}\right)$. 
Influência dos aditivos dispersantes e aditivo inorgânico

A avaliação quanto à influência de diferentes aditivos dispersantes no processo de hidratação dos ligantes puros é apresentada nas Figs. 1-5. Para os ligantes CA14M e CA270 foram observados comportamentos similares quanto à atuação desses aditivos. Em geral, os aditivos promoveram o retardamento do tempo de pega, principalmente para o teor de $0,9 \mathrm{mg} / \mathrm{m}^{2}$, quando comparado ao tempo na ausência de aditivo (em destaque nas Figs. 1 e 2).

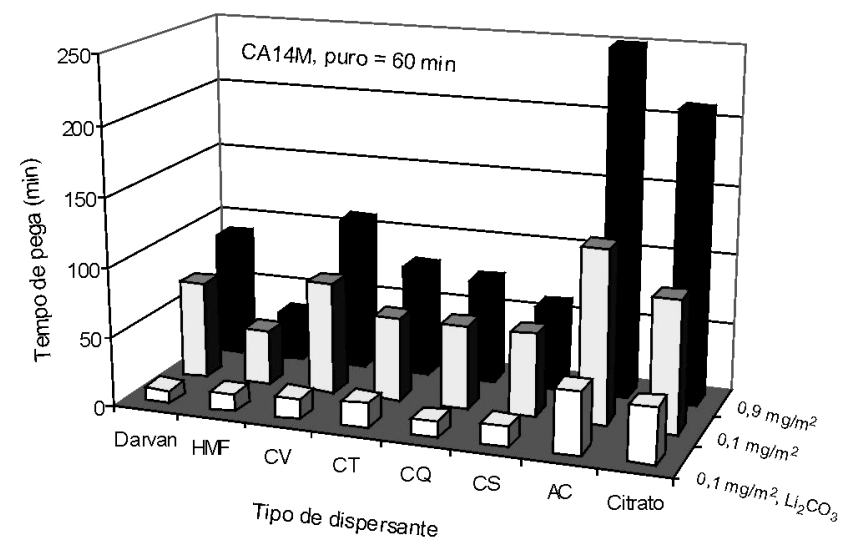

Figura 1: Tempos de pega avaliados por meio de medidas de temperatura em função do tempo, para suspensões aquosas do ligante CA14M na presença de diferentes dispersantes $\left(0,1 \mathrm{ou} 0,9 \mathrm{mg} / \mathrm{m}^{2}\right)$ ou com dispersante e $0,01 \%$ - $\mathrm{Li}_{2} \mathrm{CO}_{3}$, para a temperatura de ensaio de $50{ }^{\circ} \mathrm{C}$.

[Figure 1: Setting time evaluated through temperature measurements as a function of the time for aqueous suspensions of the CA14M binder with different dispersants $\left(0.1 \mathrm{or} 0.9 \mathrm{mg} / \mathrm{m}^{2}\right)$ or with dispersant$0.01 w t \% \mathrm{Li}_{2} \mathrm{CO}_{3}$, at $50^{\circ} \mathrm{C}$.]

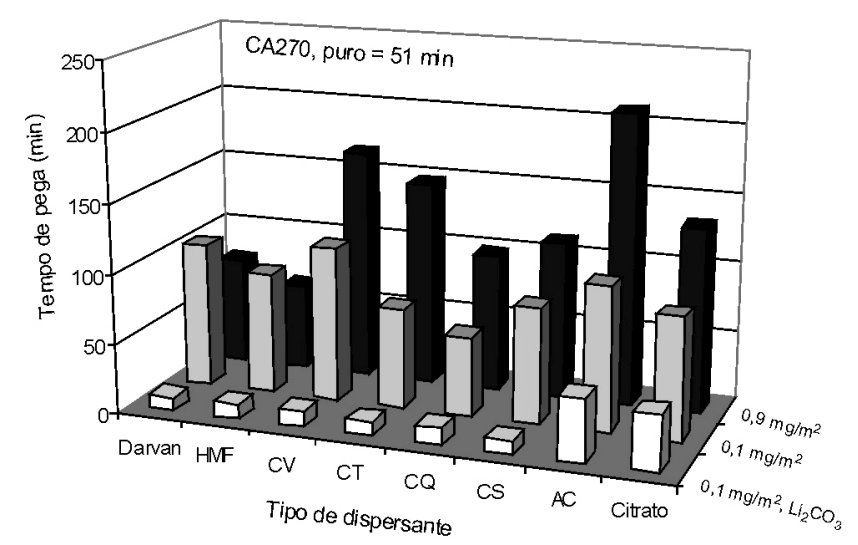

Figura 2: Tempos de pega avaliados por meio de medidas de temperatura em função do tempo, para suspensões aquosas do ligante CA270 na presença de diferentes dispersantes $\left(0,1 \mathrm{ou} 0,9 \mathrm{mg} / \mathrm{m}^{2}\right)$ ou com dispersante e $0,01 \%$-p $\mathrm{Li}_{2} \mathrm{CO}_{3}$, para a temperatura de ensaio de $50{ }^{\circ} \mathrm{C}$.

[Figure 2: Setting time evaluated through temperature measurements as a function of the time for aqueous suspensions of the CA270 binder with different dispersants $\left(0.1\right.$ or $\left.0.9 \mathrm{mg} / \mathrm{m}^{2}\right)$ or with dispersant$0.01 w t \% \mathrm{Li}_{2} \mathrm{CO}_{3}$, at $\left.50^{\circ} \mathrm{C}.\right]$
O retardamento do tempo de pega pode ser entendido visto que os ânions produzidos por esses aditivos dispersantes em solução são formados por grupos R$\mathrm{COO}^{-}$e R-OH que possuem grande afinidade pelo cálcio. A forte interação desses ânions em solução em relação ao $\mathrm{Ca}^{2+}$ tem duas conseqüências no processo de hidratação do ligante. A primeira delas consiste no fato de que a reação estequiométrica entre os ânions e o $\mathrm{Ca}^{2+}$ produz sais insolúveis em $\mathrm{pH}$ alcalino ( $\mathrm{pH}$ do meio contendo cimento) o que causa uma diminuição da relação entre as concentrações dos íons $\mathrm{Ca}^{2+}$ e $\mathrm{Al}(\mathrm{OH})_{4}{ }^{-}$. Assim, a nucleação e crescimento dos hidratos de aluminato de cálcio são mais lentos, já que a fase mais solúvel $\left(\mathrm{AH}_{3}\right)$ é favorecida [12-14].

A segunda conseqüência está relacionada ao fato de que a forte interação dos ânions gerados em solução com o cálcio possibilita a formação de sais insolúveis mesmo na interface sólido-líquido formada pela partícula do cimento e a solução. Nesse caso, a formação desses sais proporciona o surgimento de uma camada sobre a superfície das partículas de cimento. A formação dessa camada dificulta o prosseguimento da reação do cimento com a água, atrasando o estágio de saturação da solução [12-14].

Entre os diferentes tipos de aditivos retardadores, o ácido cítrico e o citrato se destacaram. Esse comportamento deve-se principalmente ao fato de que os aditivos apresentam número de sítios ionizáveis e constantes ácidas distintas, sendo que o AC e o citrato devem apresentar mais sítios ionizáveis e também as maiores constantes ácidas, produzindo assim maior número de ânions com grande afinidade pelo cálcio.

Cabe ressaltar que o ácido cítrico apresentou um superior efeito retardador quando comparado ao citrato de diamônio. O processo de nucleação de hidratos envolve a ligação entre grupos $\mathrm{OH}$ de uma molécula de aluminato de cálcio hidratado com outra molécula desse composto, por meio da sobreposição do par de elétrons isolado do oxigênio com um orbital vazio do átomo de cálcio [12]. Íons hidrogênio podem se ligar fortemente aos grupos $\mathrm{OH}$ dos hidratos bloqueando o processo de nucleação e consequentemente retardando a precipitação de hidratos. Por esse motivo, o fato do ácido cítrico apresentar mais hidrogênios ionizáveis pode explicar seu superior efeito retardador.

Quanto à combinação dos aditivos dispersantes com o acelerador $\mathrm{Li}_{2} \mathrm{CO}_{3}$, as Figs. 1 e 2 mostram que o efeito do acelerador não foi modificado pela presença dos aditivos dispersantes, como exceção para os aditivos AC e citrato. Observa-se que nestes casos ocorreu uma combinação dos efeitos retardador ( $\mathrm{AC}$ e citrato) e acelerador $\left(\mathrm{Li}_{2} \mathrm{CO}_{3}\right)$, obtendo-se um tempo de pega intermediário quando comparado ao tempo verificado quando na presença dos aditivos isolados.

Para os demais ligantes ocorreram algumas variações quanto ao comportamento relatado. No caso do Secar 71 e do Secar Plenium foi observado que os aditivos apresentaram um leve efeito retardador até mesmo para o caso do AC e citrato (Figs. 3 e 4). Isso provavelmente se deveu a razões distintas visto que o Secar 71 apresenta um maior teor de $\mathrm{CaO}$ (Tabela I) quando comparado aos cimentos CA14M e 


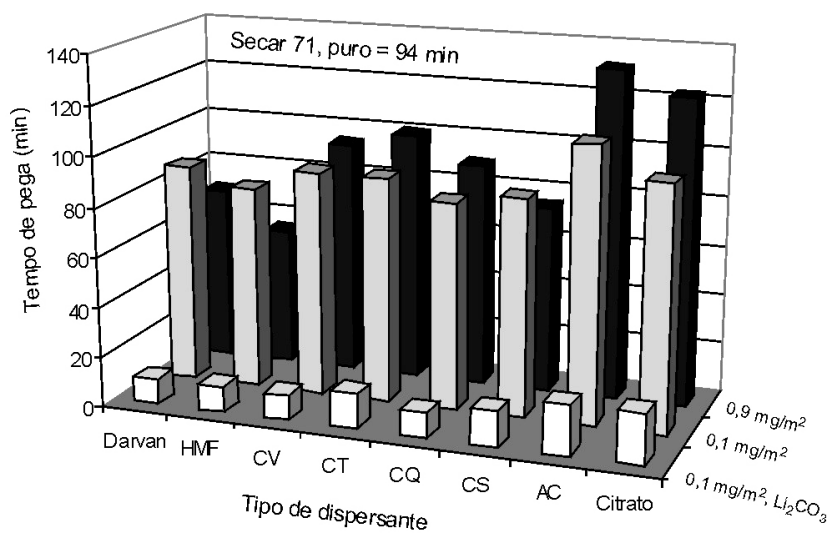

Figura 3: Tempos de pega avaliados por meio de medidas de temperatura em função do tempo, para suspensões aquosas do ligante Secar 71 na presença de diferentes dispersantes $\left(0,1\right.$ ou $\left.0,9 \mathrm{mg} / \mathrm{m}^{2}\right)$ ou com dispersante e $0,01 \%$-p $\mathrm{Li}_{2} \mathrm{CO}_{3}$, para a temperatura de ensaio de $50{ }^{\circ} \mathrm{C}$. [Figure 3: Setting time evaluated through temperature measurements as a function of the time for aqueous suspensions of the Secar 71 binder with different dispersants $\left(0.1 \mathrm{or} 0.9 \mathrm{mg} / \mathrm{m}^{2}\right)$ or with dispersant$0.01 w t \% \mathrm{Li}_{2} \mathrm{CO}_{3}$, at $50^{\circ} \mathrm{C}$.]

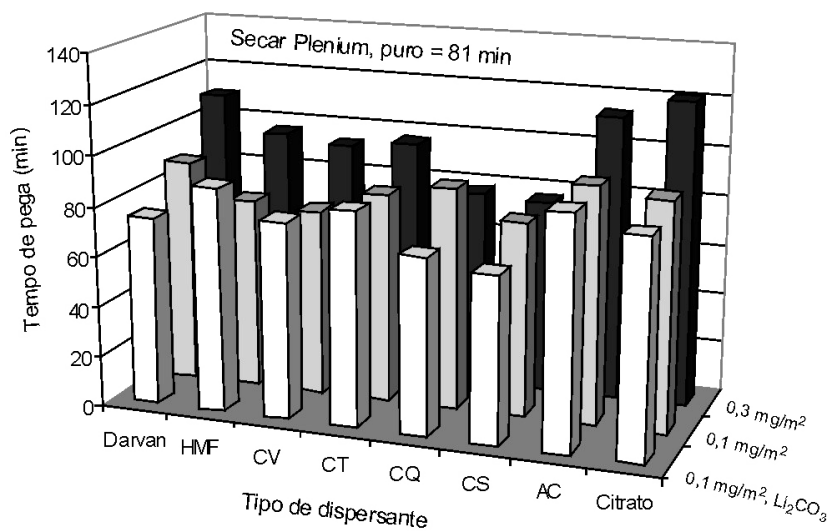

Figura 4: Tempos de pega avaliados por meio de medidas de temperatura em função do tempo, para suspensões aquosas do ligante Secar Plenium na presença de diferentes dispersantes $\left(0,1\right.$ ou $\left.0,3 \mathrm{mg} / \mathrm{m}^{2}\right)$ ou com dispersante e $0,02 \%-\mathrm{p} \mathrm{Li}_{2} \mathrm{CO}_{3}$, para a temperatura de ensaio de $50{ }^{\circ} \mathrm{C}$. [Figure 4: Setting time evaluated through temperature measurements as a function of the time for aqueous suspensions of the Secar Plenium binder with different dispersants $\left(0.1 \mathrm{or} 0.3 \mathrm{mg} / \mathrm{m}^{2}\right)$ or with dispersant$0.02 \mathrm{wt} \% \mathrm{Li}_{2} \mathrm{CO}_{3}$, at $50^{\circ} \mathrm{C}$.]

CA270 e portanto seu tempo de pega foi menos sensível ao seqüestro de íons $\mathrm{Ca}^{2+}$. Sendo assim, mesmo com o seqüestro de íons, este ligante ainda apresenta íons suficientes para formar o hidrato menos solúvel. Já o Secar Plenium, ao contrário, apresenta um reduzido teor de $\mathrm{CaO}$ (Tabela I) e portanto o hidrato com maior tendência de formação é o $\mathrm{AH}_{3}$. Dessa forma, o seqüestro de íons $\mathrm{Ca}^{2+}$ por parte dos aditivos não influencia sua formação. Também, neste caso é observado que o efeito do carbonato de lítio foi reduzido. A composição química do Secar Plenium pode explicar o reduzido efeito do acelerador como relatado anteriormente.
Para o caso do Alphabond 300 (Fig. 5) observa-se um curto tempo de reação, uma vez que as aluminas hidratáveis apresentam um processo de hidratação bem mais simples. Além disso, a alta área superficial desse ligante facilita a sua reação com a água. Neste caso, os aditivos HMF e CV também se mostraram ótimos retardadores comparáveis ao $\mathrm{AC}$ e citrato. Entretanto, somente os aditivos AC e citrato apresentaram um efeito significativo quanto à inibição do efeito do acelerador devido ao número de ânions com grande afinidade pelo cálcio apresentado por esses aditivos ser superior ao apresentado pelo HMF e CV.

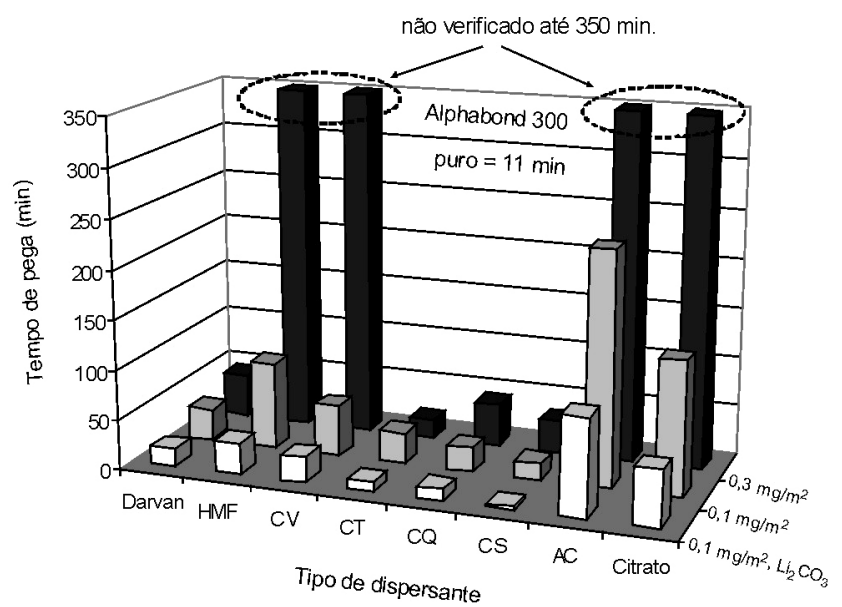

Figura 5: Tempos de pega avaliados por meio de medidas de temperatura em função do tempo, para suspensões aquosas do ligante Alphabond 300 na presença de diferentes dispersantes $\left(0,1 \mathrm{ou} 0,3 \mathrm{mg} / \mathrm{m}^{2}\right)$ ou com dispersante e $0,32 \%-\mathrm{p} \mathrm{Li}_{2} \mathrm{CO}_{3}$, para a temperatura de ensaio de $50{ }^{\circ} \mathrm{C}$. [Figure 5: Setting time evaluated through temperature measurements as a function of the time for aqueous suspensions of the Alphabond 300 binder with different dispersants $\left(0.1\right.$ or $\left.0.3 \mathrm{mg} / \mathrm{m}^{2}\right)$ or with dispersant$0.32 \mathrm{wt} \% \mathrm{Li}_{2} \mathrm{CO}_{3}$, at $50^{\circ} \mathrm{C}$.]

\section{Efeito combinado de aditivos na presença de matriz}

$\mathrm{O}$ efeito combinado de aditivos foi também verificado em suspensões de ligantes na presença da matriz Esy-Pump por meio de ensaios oscilatórios.

Nesse tipo de ensaio, a tensão aplicada obedece a uma função senoidal do tempo e os dados de viscosidade e elasticidade gerados são relacionados à freqüência aplicada. Esse método é especialmente adequado para sistemas que, por meio de um processo físico ou químico, gradualmente passam do estado líquido para o sólido. Também apresenta a vantagem de poder utilizar deformações tão pequenas que não alterem significativamente o sistema [15].

As medidas do módulo de armazenamento (G') permitem verificar a influência do fenômeno de hidratação nas interações coloidais e comportamento de pega inicial. Com a consolidação da suspensão é verificado um aumento de G'.

Pode ser observado na Fig. 6 que esses ensaios confirmam os efeitos acelerador, retardador e intermediário dos aditivos $\mathrm{Li}_{2} \mathrm{CO}_{3}, \mathrm{AC}$ e $\mathrm{AC}-\mathrm{Li}_{2} \mathrm{CO}_{3}$, respectivamente. Também, no 

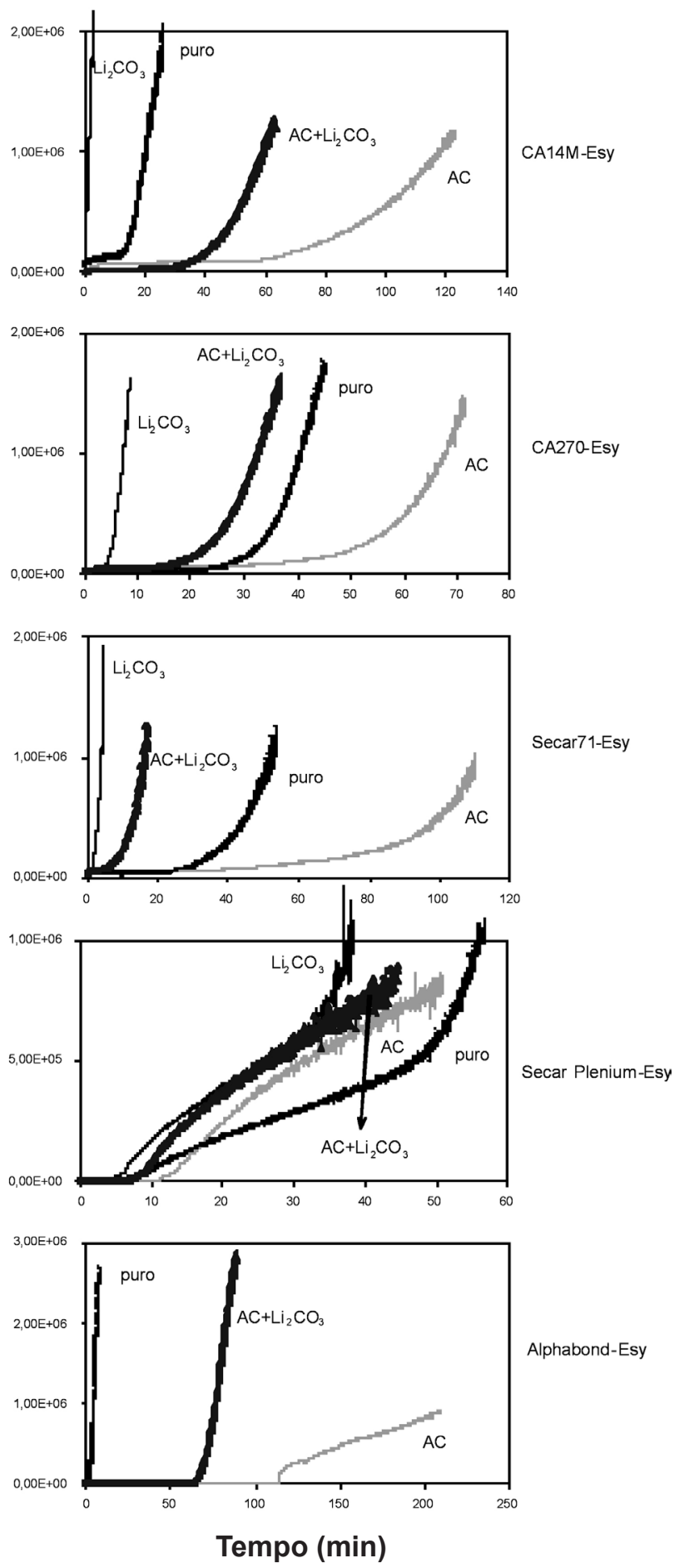

Figura 6: Módulos de armazenamento (G') a $50^{\circ} \mathrm{C}$ em função do tempo para suspensões dos ligantes CA14M, CA270, Secar 71, Secar Plenium e Alphabond 300 na presença de matriz Esy Pump $\left(50: 50 \%\right.$-p) e dos aditivos ácido cítrico $\left(0,1 \mathrm{mg} / \mathrm{m}^{2}\right)$ e $\mathrm{Li}_{2} \mathrm{CO}_{3}$ $(0,01 \%$-p ou $0,02 \%$-p para o Secar Plenium e $0,32 \%$-p para Alphabond 300) quando puros ou combinados.

[Figure 6: Storage moduli $\left(G^{\prime}\right)$ at $50^{\circ} \mathrm{C}$ as a function of the time for CA14M, CA270, Secar 71, Secar Plenium e Alphabond 300 binders suspensions in the presence of Esy Pump matrix (50:50 $w t \%)$ and of citric acid $\left(0.1 \mathrm{mg} / \mathrm{m}^{2}\right)$ and $\mathrm{Li}_{2} \mathrm{CO}_{3}(0.01 \mathrm{wt} \%$ or 0.02 $w t \%$ for Secar Plenium and $0.32 \mathrm{wt} \%$ for Alphabond 300) additives for plain and combined systems.] caso do Secar Plenium não foram observadas diferenças significativas como previsto pelos ensaios de temperatura.

Entretanto, os ensaios reológicos oscilatórios se mostram particularmente importantes na determinação da ocorrência da consolidação de suspensões dos ligantes visto que ocorre uma defasagem no tempo de consolidação de suspensões sob condições idênticas quando medido por meio de ensaios de temperatura e oscilatórios [16].

Tal comportamento pode ser entendido visto que tão logo os cristais de hidratos começam a precipitar (início do aumento de temperatura), eles crescem a partir das partículas de alumina ocorrendo o entrelaçamento deles devido a reduzida distância entre as partículas, o que resulta no aumento de G' (consolidação da suspensão), mesmo antes de se atingir o máximo de temperatura. Isso pode explicar porque é verificado um tempo de pega tão rápido quando medido por meio dos ensaios reológicos [16]. Portanto, o ensaio de temperatura pode ser correlacionado diretamente com o processo de hidratação do cimento enquanto o ensaio oscilatório com a consolidação da suspensão, ou seja, os ensaios são sensíveis a fenômenos distintos. Desse modo, pode-se concluir que o ensaio oscilatório pode determinar mais precisamente a trabalhabilidade da suspensão.

Entretanto, embora o aumento de G' indique um aumento da consistência da suspensão, um ensaio adicional faz-se necessário para indicar o tempo de desmoldagem $[6,9]$.

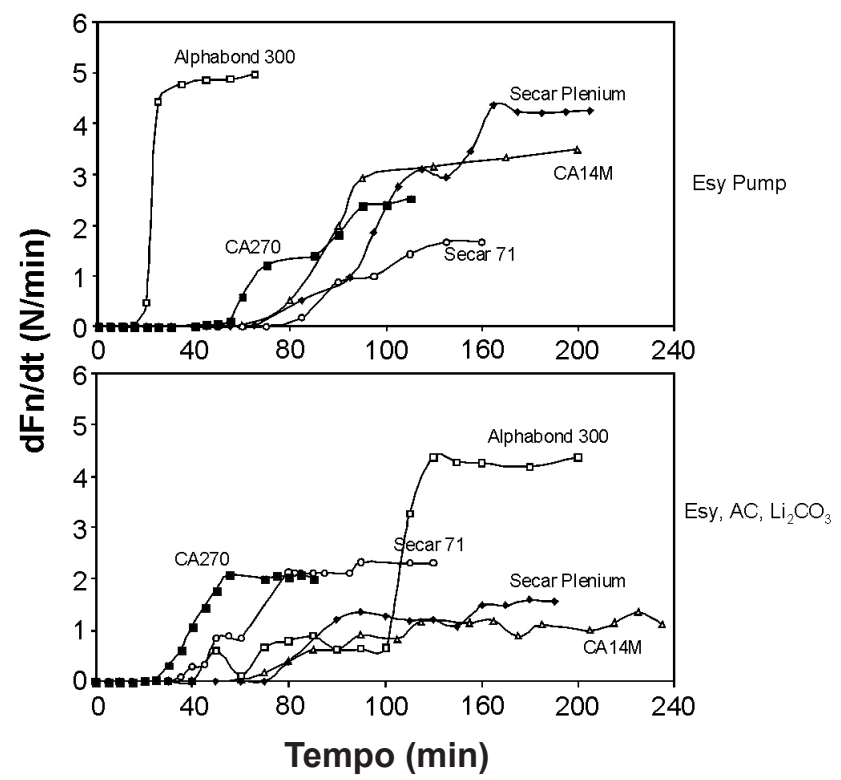

Figura 7: Taxa de força normal $(\mathrm{dFn} / \mathrm{dt})$ a $50{ }^{\circ} \mathrm{C}$ em função do tempo para suspensões dos ligantes CA14M, CA270, Secar 71, Secar Plenium e Alphabond 300 na presença de matriz Esy Pump (50:50\%-p) e Esy Pump+ácido cítrico $\left(0,1 \mathrm{mg} / \mathrm{m}^{2}\right)+\mathrm{Li}_{2} \mathrm{CO}_{3}(0,01 \%$-p ou 0,02\%-p para o Secar Plenium e 0,32\%-p para Alphabond 300). [Figure 7: Normal force rate (dFn/dt) at $50^{\circ} \mathrm{C}$ as a function of the time for CA14M, CA270, Secar 71, Secar Plenium e Alphabond 300 binders suspensions in the presence of Esy Pump matrix (50:50 wt\%) and Esy Pump+citric acid $\left(0.1 \mathrm{mg} / \mathrm{m}^{2}\right)+\mathrm{Li}_{2} \mathrm{CO}_{3}(0.01 \mathrm{wt} \%$ or 0.02 $w t \%$ for Secar Plenium and 0.32 wt\% for Alphabond 300).] 
Dessa forma, para os sistemas ligante-Esy e liganteEsy-aditivos (Fig. 7) foi determinado em que momento as suspensões apresentaram a máxima resistência à deformação imposta. Observa-se que em todos os casos a taxa de força normal aumenta com o tempo, indicando aumento da resistência à deformação da suspensão imposta pela ação do ligante. Para os diferentes ligantes a ordem quanto ao tempo de desmoldagem foi a mesma verificada quanto a trabalhabilidade, ou seja, o ligante Alphabond 300 promoveu a consolidação no menor tempo enquanto para o Secar Plenium o aumento da taxa de força normal foi o mais lento. Assim como nos ensaios reológicos o tempo de desmoldagem sofre um deslocamento, quando da presença de aditivos, para maior ou menor tempo de acordo com a predominância dos efeitos dos aditivos. No caso do Alphabond $300 \mathrm{o}$ efeito do retardador é predominante deslocando o tempo de desmoldagem de 40 (sem aditivo) para 140 minutos. Para o CA270 o efeito do acelerador predomina verificando-se deslocamento de 120 para 60 minutos enquanto no Secar Plenium não houve alteração significativa.

\section{CONCLUSÕES}

A presença de matriz promove redução do tempo de pega, devido as suas partículas constituir sítios de nucleação facilitando a precipitação de hidratos. No entanto, tal redução do tempo de pega pode ser principalmente atribuída ao teor de $\mathrm{Na}_{2} \mathrm{O}$ das diferentes matrizes. $\mathrm{O}$ aumento do sódio liberado em solução favorece a precipitação do hidrato menos solúvel uma vez que diminui a quantidade de íons $\mathrm{Al}(\mathrm{OH})_{4}^{-}$por meio da formação de $\mathrm{NaAl}(\mathrm{OH})_{4}$.

O mesmo comportamento é verificado na presença do aditivo carbonato de lítio, ou seja, a presença de $\mathrm{Li}^{+}$em solução proporciona a formação de compostos insolúveis do tipo $\mathrm{LiAl}(\mathrm{OH})_{4}$, retirando íons $\mathrm{Al}(\mathrm{OH})_{4}^{-}$da solução. A precipitação desses compostos é mais rápida do que a precipitação dos hidratos de aluminato de cálcio e apresenta estrutura cristalina necessária para servir de germes para a nucleação do hidrato $\mathrm{C}_{2} \mathrm{AH}_{8}$, acelerando a pega dos cimentos. Entretanto, o efeito acelerador do $\mathrm{Li}_{2} \mathrm{CO}_{3}$ é reduzido na presença de matriz.

Para os ligantes CA14M, CA270 e Alphabond 300 os aditivos ácido cítrico e citrato de diamônio apresentaramse como os mais eficientes em promover o retardamento do tempo de pega por possuírem mais sítios ionizáveis e também as maiores constantes ácidas, produzindo assim maior número de ânions com grande afinidade pelo cálcio. $\mathrm{O}$ seqüestro dos íons $\mathrm{Ca}^{2+}$ pelos seus ânions diminui a relação entre as concentrações dos íons provenientes da dissolução, resultando na precipitação do hidrato mais solúvel. Já para o Secar 71 e o Secar Plenium tais aditivos apresentaram um leve efeito retardador.

Os ensaios oscilatórios e de força normal confirmaram os efeitos apresentados pela matriz e aditivos quando isolados ou combinados mostrando-se úteis na determinação da ocorrência da consolidação das suspensões (tempo limite para a moldagem) e evolução da resistência mecânica dos corpos conformados (tempo mínimo para a desmoldagem).

\section{AGRADECIMENTOS}

À Almatis pelo fornecimento das matérias-primas, a SKW Polymers pelo fornecimento de aditivos e à FAPESP pelo suporte financeiro.

\section{REFERÊNCIAS}

[1] C. M. George, "Aspects of calcium aluminate cement hydration", in Refractories Symposium, The American Ceramic Society, St. Louis (1994) 1-21.

[2] J. R. Garcia, I. R. Oliveira, V. C. Pandolfelli, "Processo de hidratação e os mecanismos de atuação dos aditivos aceleradores e retardadores de pega do cimento de aluminato de cálcio", Cerâmica 53, 325 (2006) 42-56.

[3] C. D. Parr, C. Aalt, C. Wohrmeyer, E. Nonnet, "Calcium aluminate cement hydration and its influence on the placing and high temperature properties of reduced cement castable systems", in Proc. $39^{\text {th }}$ Annual Symp. Refractories, St Louis, April 9-10, 2003.

[4] W. Ma, P. W. Brown, "Mechanisms of reaction of hydratable aluminas", J. Am. Ceram. Soc. 82, 2 (1999) 453456.

[5] Y. Hongo, " $\rho$-Alumina bonded castable refractories", Taikabutsu Overseas 9, 1 (1988) 35-38.

[6] I. R. Oliveira, R. D. Anjos, V. C. Pandolfelli, "Técnicas para avaliação da consolidação de suspensões cerâmicas com o uso de diferentes ligantes", Cerâmica 53, 326 (2007) 133-141.

[7] G, Schramm, "A practical approach to rheology and rheometry", $2^{\text {nd }}$ Ed., Karlsruhe: Gebrueder HAAKE GmbH, (1998) $291 \mathrm{p}$.

[8] C. Servais, "Rheological methods for multiphase materials, in Int. Symp. Food Rheol. Struct. 3., Zurich (2003) 137-141.

[9] R. D. dos Anjos, R. Salomão, V. C. Pandolfelli, "Novas técnicas de reometria aplicadas a suspensões cerâmicas", Cerâmica 51, 315 (2005) 173-179.

[10] I. R. Oliveira, J. R. Garcia, V. C. Pandolfelli, "Cinética de hidratação de ligantes a base de alumina hidratável ou aluminato de cálcio", Cerâmica 53, 325 (2007) 20-28.

[11] W. Gebner, S. Mohmel, A. Schmalstieg, A. Rettel, J. P. Bayoux, A. Capmas, A. Mathieu, "On the influence of the specific surface area and $\mathrm{Na}_{2} \mathrm{O}$ content of aluminas on the hydration processes in $\mathrm{CaO} \cdot \mathrm{Al}_{2} \mathrm{O}_{3} / \mathrm{Al}_{2} \mathrm{O}_{3}$ Mixes", in Proc. UNITECR 95 (Unified International Conference on Refractories), Kyoto (1995) 313-320.

[12] B. R. Currell, R. Grzeskowlak, H. G. Midgley, J. R. Parsonage, "The acceleration and retardation of set high alumina cement by additives", Cement and Concrete Research 7 (1987) 420-432.

[13] N. Bunt, C. Revais, M. Vialle, "Additives in calcium aluminate cement containing castables", in Proc. UNITECR 97 (Unified International Technical Conference on Refractories), New Orleans (1997) 1347-1354. 
[14] S. A. Rodger, D. D. Double, "The chemistry of hydration of high alumina cement in the presence of accelerating and retarding admixtures", Cement and Concrete Research $\mathbf{1 4}$ (1984) 73-82.

[15] G. W. S. Blair, "Elementary rheology", London,
Academic Press Inc. (1969)158 p.

[16] I. R. Oliveira, J. R. Garcia, V. C. Pandolfelli, "Influência de aditivos dispersantes e acelerador na hidratação de cimento e cimento-matriz", Cerâmica 52, 323 (2005)184-193.

(Rec. 21/10/2006, Ac. 31/11/2006) 